Bangladesh J. Plant Taxon. 25(2): 273-288, 2018 (December)

(C) 2018 Bangladesh Association of Plant Taxonomists

\title{
FLORISTIC DIVERSITY (MAGNOLIIDS AND EUDICOTS) OF BARAIYADHALA NATIONAL PARK, CHITTAGONG, BANGLADESH
}

\author{
Mohammad Harun-ur-Rashid ${ }^{1}$, Saiful Islam and Sadia Binte Kashem \\ Department of Botany, University of Chittagong, Chittagong 4331, Bangladesh
}

Keywords: Plant diversity; Baraiyadhala National Park; Conservation management.

\begin{abstract}
An intensive floristic investigation provides the first systematic and comprehensive account of the floral diversity of Baraiyadhala National Park of Bangladesh, and recognizes 528 wild taxa belonging to 337 genera and 73 families (Magnoliids and Eudicots) in the park. Habit analysis reveals that trees (179 species) and herbs (174 species) constitute the major categories of the plant community followed by shrubs (95 species), climbers (78 species), and two epiphytes. Status of occurrence has been assessed for proper conservation management and sustainable utilization of the taxa resulting in $165(31.25 \%)$ to be rare, $23(4.36 \%)$ as endangered, $12(2.27 \%)$ as critically endangered and 4 species $(0.76 \%)$ are found as vulnerable in the forest. Fabaceae is the dominant family represented by 75 taxa, followed by Rubiaceae (47 taxa), Malvaceae (28 species), Asteraceae (27 species) and Euphorbiaceae ( 24 species). Twenty-three families represent single species each in the area.
\end{abstract}

\section{Introduction}

Baraiyadhala National Park as one of the important Protected Areas (PAs) of Bangladesh that lies between $22^{\circ} 40.489^{\prime}-22^{\circ} 48^{\prime} \mathrm{N}$ latitude and $90^{\circ} 40^{\prime}-91^{\circ} 55.979^{\prime} \mathrm{E}$ longitude and located in Sitakundu and Mirsharai Upazilas of Chittagong district. The forest is under the jurisdiction of Baraiyadhala Forest Range of Chittagong North Forest Division. The park encompasses 2,933.61 hectare $(7,249$ acres) area and is classified under Category II of the International IUCN classification of protected areas (Hossain, 2015). Formerly, it was a part of the Reserved Forest of Chittagong North Forest Division, and was declared as Baraiyadhala National Park on 6th April, 2010 by the Ministry of Environment and Forest through a Gazzette notification no. MoEF/ForSec-02/02 National Park/10/2010/210 dated 06/04/2010 under the Provisions of Article 23(1) of the Bangladesh Wildlife (Preservation) (Amendment) Act, 1974; which has now been altered by Wildlife (Conservation and Security) Act, 2012 (Hossain, 2015). The park comprises three blocks: Baraiyadhala and Wahidpur Blocks under Baraiyadhala Forest Beat, and Kunderhat Block under Bartakia Forest Beat.

The landscape of the forest is characterized by hills, valleys, gullies and numerous water streams and covered mainly by secondary degraded forests and plantations. Floristically Baraiyadhala National Park is rich and diverse. Hence, it is very important to take proper steps to conserve this natural forest; and to explore, document and analyze the species diversity occurring in the Baraiyadhala National Park before disappearing from nature.

However, the forest has not been botanized to determine the plant species diversity, their status of occurrence and conservation measures. Therefore, the present investigation aims to explore, collect, and document the Angiospermic (Magnoliids and Eudicots) plant resources in the forest, their conservation management and sustainable utilization.

\footnotetext{
${ }^{1}$ Corresponding author. Email: haruncu@gmail.com
} 


\section{Material and Methods}

The survey of the flora has been made through repeated field visits during February 2016 to July 2017 in Baraiyadhala National Park. Random sampling and collections of fertile specimens have been made for identification and voucher specimens have been preserved at Herbarium of Chittagong University (HCU). Collected specimens have been critically examined, studied and identified. Identifications have been confirmed by consulting standard literature and specimens and taxonomists of HCU and Bangladesh National Herbarium (DACB).

Nomenclature has been updated following recent literature (Ahmed et al., 2008, 2009a,b,c,d; Rashid and Rahman, 2011, 2012), and confirmed with consulting The Plant List (2013) (http://www.theplantlist.org). Families are arranged according to the classification of Angiosperm Phylogeny Group (APG IV, 2016). The taxa are listed alphabetically under each family along with their habit, Bangla name, and status of occurrence (Table 1). Local names of many plants have been noted from local people during field trips and/or consulting Prain (1903), Heinig (1926), Huq (1986), Das and Alam (2001), and Dey et al. (1999).

\section{Results and Discussion}

The present inventory provides the first systematic and comprehensive account of the floral diversity of the forest and recognizes 528 wild taxa belonging to 337 genera and 73 families (Magnoliids and Eudicots) in the park (Table 1).

Baraiyadhala National Park presents diverse habitat including hills, valleys, gullies and numerous water streams and covered mainly by secondary degraded forests. Some patches of the forest are planted with Acacia auriculiformis (Akashmoni), Artocarpus chama (Chapalish), Chukrasia tabularis (Chikrassi), Eucalyptus sp. (Eucalyptus), Gmelina arborea (Gamar), Dipterocarpus turbinatus (Garjan), Swietenia mahagoni (Mehogini), Azadirachta indica (Neem), Aphanamixis polystachya (Pitraj), Tectona grandis (Segun), Hopea odorata (Telsur), Toona ciliata (Toon) etc. The palms, rattans and bamboos mostly occupy the valleys. Common shrubs, herbs, grasses and babanas are fragmented to degraded habitats. A few individuals of Boilam (Anisoptera scaphula), Civit (Swintonia floribunda) and Lohakat (Xylia xylocarpa var. kerrii) are still available as characteristic elements of the forest. Some epiphytic species of Cymbidium, Dendrobium, Drynaria, Raphidophra, and Phothos are distributed in the forest area, while luxuriant growth of Aroids, Begonias, Bryophytes and Pteridophytes is observed in the natural moist habitats of the park. One of the most characteristic features of this forest is the occurrence of three indigenous gymnospermic species, Cycas pectinata Buch.-Ham., Podocarpus neriifolius D. Don and Gnetum montanum Markgr. In Bangladesh, Baraiyadhala National Park is the only home of $C$. pectinata. The biodiversity of the area is highly imperiled due to anthropogenic activities, including habitat destruction, over-exploitation, unsustainable hunting and all of these three species are critically endangered and are on the verge of extinction in the forest. However, Nguyen (2010) categorizes C. pectinata as Vulnerable (VU).

The park is dominated by trees and herbs consisting of 179 (33.90\%) and 174 (32.95\%) species respectively, followed by $18 \%$ shrub (95 spp.), $14.77 \%$ climbers (78 spp.), and two epiphytes. Fabaceae appears as the largest family with 75 taxa, followed by Rubiaceae ( 47 taxa), Malvaceae (28 spp.), Asteraceae (27 spp.) and Euphorbiaceae ( 24 spp.). Twenty-three families are represented by single species each in the study area. Ten dominant families (Fig. 2) constitute 320 species amounting $60.60 \%$ of the total species reported from the park, while remaining 63 families comprise only $39.40 \%$ of total species.

Status of occurrence has been assessed for proper conservation management and sustainable utilization of the natural resources of the forest. A total of 165 species $(31.25 \%)$ are found to be rarely distributed in the forest, while $23(4.36 \%)$ are assessed as endangered, $12(2.27 \%)$ as 
Table 1. Plant diversity in Baraiyadhala National Park.

\begin{tabular}{|c|c|c|c|c|}
\hline Family & Taxa & Bangla name & Habit & $\begin{array}{l}\text { Status of } \\
\text { occurrence }\end{array}$ \\
\hline \multirow[t]{4}{*}{ Piperaceae } & Peperomia pellucida (L.) Kunth & Luchi pata & $\mathrm{H}$ & Common \\
\hline & Piper longum L. & Pipul & $\mathrm{H}$ & Rare \\
\hline & P. rhytidocarpum Hook. f. & Ban pipul & $\mathrm{CS}$ & Common \\
\hline & P. sylvaticum Roxb. & Ban Pan & $\mathrm{Cr}$ & Common \\
\hline \multirow[t]{3}{*}{ Aristolochiaceae } & Aristolochia indica $\mathrm{L}$. & Ishwarmul & $\mathrm{Cl}$ & Rare \\
\hline & A. saccata Wall. & Ishwarmul & $\mathrm{Cl}$ & Rare \\
\hline & A. tagala Cham. & Ishwarmul & WT & Rare \\
\hline Myristicaceae & $\begin{array}{l}\text { Knema erratica (Hook. f. \& Thom.) } \\
\text { Sinclair }\end{array}$ & - & $\mathrm{T}$ & $\begin{array}{l}\text { Critically } \\
\text { endangered }\end{array}$ \\
\hline Magnoliaceae & $\begin{array}{l}\text { Magnolia champaca (L.) Baill. ex } \\
\text { Pierre }\end{array}$ & Champa & $\mathrm{T}$ & Common \\
\hline \multirow[t]{8}{*}{ Annonaceae } & $\begin{array}{l}\text { Artabotrys caudatus Wall. ex Hook. f. } \\
\& \text { Thomson }\end{array}$ & - & WC & Endangered \\
\hline & Desmos chinensis Lour. & Sotoyalang & $\mathrm{S}$ & Common \\
\hline & D. dumosus (Roxb.) Saff. & - & WC & Endangered \\
\hline & $\begin{array}{l}\text { Fissistigma rubiginosum (A. DC.) } \\
\text { Merr. }\end{array}$ & - & WC & Endangered \\
\hline & F. wallichii (Hook.f. \& Thom.) Merr. & - & WC & Endangered \\
\hline & Uvaria dioeca Roxb. & Tasbi & $\mathrm{T}$ & Vulnerable \\
\hline & U. hamiltonii Hook. f. \& Thom. & Latkan & WC & Endangered \\
\hline & U. littoralis (Blume) Blume & Bagh-runga & WC & Vulnerable \\
\hline \multirow[t]{4}{*}{ Lauraceae } & $\begin{array}{l}\text { Actinodaphne gullavara (Buch.-Ham. } \\
\text { ex Nees) Almeida }\end{array}$ & Tejmatan & $\mathrm{T}$ & Common \\
\hline & Litsea glutinosa (Lour.) C.B. Rob. & Menda & $\mathrm{T}$ & Common \\
\hline & L. monopetala (Roxb.) Pers. & Menda & $\mathrm{T}$ & Rare \\
\hline & Machilus gamblei King ex Hook. f. & Nala-amsi & $\mathrm{T}$ & Rare \\
\hline \multirow[t]{6}{*}{ Menispermaceae } & Cocculus hirsutus (L.) Theob. & Jaljamani & & Rare \\
\hline & Parabaena sagittata Miers. & Jaljamani & $\mathrm{Cl}$ & Common \\
\hline & Stephania glabra (Roxb.) Miers & Musahanilata & WC & Rare \\
\hline & S. japonica (Thunb.) Miers & Nimukha & WC & Common \\
\hline & S. reticulata Forman & - & $\mathrm{Cl}$ & Common \\
\hline & $\begin{array}{l}\text { Tinospora crispa (L.) Hook. f. \& } \\
\text { Thom. }\end{array}$ & Gulancha & WC & Endangered \\
\hline Sabiaceae & Meliosma pinnata (Roxb.) Maxim. & Attalia & $\mathrm{T}$ & Rare \\
\hline Proteaceae & Helicia excelsa (Roxb.) Blume & - & $\mathrm{T}$ & Rare \\
\hline \multirow[t]{2}{*}{ Dilleniaceae } & Dillenia pentagyna Roxb. & Banchalta & $\mathrm{T}$ & $\begin{array}{l}\text { Critically } \\
\text { Endangered }\end{array}$ \\
\hline & D. scabrella (D. Don) Roxb. ex Wall. & Ajuli & $\mathrm{T}$ & Common \\
\hline \multirow[t]{9}{*}{ Vitaceae } & Ampelocissus barbata (Wall.) Planch. & Jarila-lahari & CS & Common \\
\hline & Cayratia japonica (Thunb.) Gagnep. & - & $\mathrm{Cl}$ & Common \\
\hline & C. trifolia (L.) Domin & Amal lata & $\mathrm{Cl}$ & Common \\
\hline & $\begin{array}{l}\text { Cissus assamica (M.A. Lawson) } \\
\text { Craib }\end{array}$ & Amasha-lata & WC & Common \\
\hline & C. elongata Roxb. & Dhemna & $\mathrm{Cl}$ & Common \\
\hline & C. javana DC. & Bichitra-lata & $\mathrm{Cl}$ & Common \\
\hline & C. pentagona Roxb. & Sona-lota & $\mathrm{Cl}$ & Rare \\
\hline & Leea aequata $\mathrm{L}$. & Kakjangha & $\mathrm{S}$ & Rare \\
\hline & L. asiatica (L.) Ridsdale & Mach & $\mathrm{S}$ & Rare \\
\hline
\end{tabular}


Table 1 Contd.

\begin{tabular}{|c|c|c|c|c|}
\hline Family & Taxa & Bangla name & Habit & $\begin{array}{l}\text { Status of } \\
\text { occurrence }\end{array}$ \\
\hline \multirow[t]{7}{*}{ Vitaceae } & L. guineensis G. Don & Phupharia & $S$ & Rare \\
\hline & L. indica (Burm.f.) Merr. & Kurkurji & $\mathrm{T}$ & Common \\
\hline & L. macrophylla Roxb. ex Hornem. & - & $\mathrm{T}$ & Rare \\
\hline & Tetrastigma angustifolia (Roxb.) Deb & Nekung riubi & $\mathrm{Cl}$ & Rare \\
\hline & T. bracteolatum (Wall.) Planch. & Golgoli lata & $\mathrm{Cl}$ & Rare \\
\hline & T. hookeri (M.A. Lawson) Planch. & Horina-lata & WC & Common \\
\hline & T. leucostaphylum (Dennst.) Alston & Nekung & WC & Rare \\
\hline \multirow[t]{38}{*}{ Fabaceae } & Abrus precatorius $\mathrm{L}$. & Kunch & WC & Common \\
\hline & A. pulchellus Wall. ex Thwaites & Kaichagula & TS & Rare \\
\hline & Adenanthera pavonina $\mathrm{L}$. & Raktachandan & $\mathrm{T}$ & Common \\
\hline & Albizia odoratissima (L. f.) Benth. & Tetuya Koroi & $\mathrm{T}$ & Common \\
\hline & A. richardiana (Voigt) King \& Prain & Gagan Siris & $\mathrm{T}$ & Common \\
\hline & A. saman (Jacq.) Merr. & Rendi koroi & $\mathrm{T}$ & Common \\
\hline & Bauhinia acuminata $\mathrm{L}$. & Sada kancon & $\mathrm{S}$ & Common \\
\hline & B. purpurea $\mathrm{L}$. & Devakanchan & $\mathrm{T}$ & Common \\
\hline & B. scandens $\mathrm{L}$. & Gendi lata & WC & Common \\
\hline & B. variegata $\mathrm{L}$. & Raktakancan & $\mathrm{T}$ & Common \\
\hline & Butea monosperma (Lam.) Taub. & Palash & $\mathrm{T}$ & Common \\
\hline & Caesalpinia bonduc (L.) Roxb. & Natakaranga & SS & Rare \\
\hline & C. digyna Rottler & Umulkuchi & $\mathrm{S}$ & Rare \\
\hline & Cajanus scarabaeoides (L.) Thouars & - & $\mathrm{Tw}$ & Rare \\
\hline & Calliandra umbrosa (Wall.) Benth. & Chotobetmara & Tree & Common \\
\hline & Cassia fistula $\mathrm{L}$. & Sonalu & Tree & Common \\
\hline & $\begin{array}{l}\text { C. javanica subsp. nodosa (Roxb.) K. } \\
\text { Larsen \& S.S. Larsen }\end{array}$ & Bon-sonalu & Tree & Rare \\
\hline & C. obtusifolia $\mathrm{L}$. & Chakunda & $\mathrm{U}$ & Common \\
\hline & Codariocalyx gyroides (Link) Hassk. & - & $\mathrm{S}$ & Rare \\
\hline & C. motorius (Houtt.) H.Ohashi & Gorachand & S & Rare \\
\hline & $\begin{array}{l}\text { Crotalaria acicularis Buch.-Ham. ex } \\
\text { Benth. }\end{array}$ & - & $\mathrm{H}$ & Common \\
\hline & C. albida Roth & - & $\mathrm{H}$ & Common \\
\hline & C. bracteata $\mathrm{DC}$ & - & $\mathrm{S}$ & Common \\
\hline & C. calycina Schrank & - & $\mathrm{H}$ & Common \\
\hline & C. dubia Graham & - & $\mathrm{H}$ & Common \\
\hline & C. ferruginea Benth. & - & $\mathrm{H}$ & Common \\
\hline & C. incana $\mathrm{L}$. & Chotojhunjhuna & $\mathrm{H}$ & Rare \\
\hline & C. pallida Aiton & Jhun-jhuni & $\mathrm{H}$ & Common \\
\hline & C. tetragona Roxb. ex Andrews & - & $\mathrm{H}$ & Rare \\
\hline & C. verrucosa $\mathrm{L}$. & Bansan & $\mathrm{U}$ & Common \\
\hline & Dalbergia sericea G. Don & Sristi & $\mathrm{T}$ & Rare \\
\hline & D. lanceolaria L. f. & Chakemdia & $\mathrm{T}$ & Rare \\
\hline & D. malabarica Prain & - & $\mathrm{T}$ & Rare \\
\hline & D. spinosa Roxb. & Ananta kantha & LS & Common \\
\hline & D. stipulacea Roxb. & Dadbari & $\mathrm{T}$ & Rare \\
\hline & D. volubilis Roxb. & Ankilata & WC & Rare \\
\hline & $\begin{array}{l}\text { Dendrolobium triangulare (Retz.) } \\
\text { Schindl. }\end{array}$ & Bir Jarwar & $\mathrm{S}$ & Rare \\
\hline & Derris robusta (DC.) Benth. & Jangaria & $\mathrm{T}$ & Rare \\
\hline
\end{tabular}


Table 1 Contd.

\begin{tabular}{|c|c|c|c|c|}
\hline Family & Taxa & Bangla name & Habit & $\begin{array}{l}\text { Status of } \\
\text { occurrence }\end{array}$ \\
\hline \multirow[t]{37}{*}{ Fabaceae } & D. scandens (Roxb.) Benth. & Amkurchi & WC & Common \\
\hline & Desmodium gangeticum (L.) DC. & Salpani & $\mathrm{S}$ & Common \\
\hline & D. heterocarpon (L.) DC. & - & S & Common \\
\hline & D. heterophyllum (Willd.) DC. & Bonmotorshuty & $\mathrm{H}$ & Common \\
\hline & D. laxiflorum DC. & - & $\mathrm{S}$ & Common \\
\hline & D. triflorum (L.) DC. & Kulalia & $\mathrm{H}$ & Common \\
\hline & Entada gigas (L.) Fawc. \& Rendle & Gila & $\mathrm{Cl}$ & Rare \\
\hline & E. rheedii Spreng. & Gilalata & WC & Rare \\
\hline & Erythrina variegata $\mathrm{L}$ & Mandar & $\mathrm{T}$ & Rare \\
\hline & Flemingia macrophylla (Willd.) Merr. & Bara salphan & LS & Common \\
\hline & F. strobilifera $($ L.) W.T.Aiton & Sim busak & $\mathrm{S}$ & Common \\
\hline & Gliricidia sepium (Jacq.) Walp. & $\begin{array}{l}\text { Bashantamanju } \\
\text { ri }\end{array}$ & MT & Rare \\
\hline & Mimosa diplotricha Sauvalle & Bara lajjabati & CS & Common \\
\hline & M. himalayana Gamble & Jharua & $\mathrm{CS}$ & Rare \\
\hline & M. pudica L. & Lajja bati & $\mathrm{H}$ & Common \\
\hline & Mucuna monosperma Wight & Soash guri & WC & Rare \\
\hline & M. pruriens (L.) DC. & Al-kushi & $\mathrm{Cl}$ & Common \\
\hline & Phyllodium pulchellum (L.) Desv. & Jatsalpani & $\mathrm{S}$ & Common \\
\hline & Pithecellobium jiringa (Jack) Merr. & $\begin{array}{l}\text { Kuramara } \\
\text { Gach }\end{array}$ & $\mathrm{S}$ & Common \\
\hline & Pueraria tuberosa (Willd.) DC. & Shimia & $\mathrm{H}$ & Common \\
\hline & $\begin{array}{l}\text { P. phaseoloides var. subspicata } \\
\text { (Benth.) Maesen }\end{array}$ & - & $\mathrm{H}$ & Common \\
\hline & Saraca asoca (Roxb.) Willd. & Ashok & $\mathrm{T}$ & Rare \\
\hline & S. indica L. & Ashok & $\mathrm{T}$ & Common \\
\hline & Senna alata (L.) Roxb. & Dad mardon & $\mathrm{S}$ & Common \\
\hline & S. occidentalis (L.) Link & $\begin{array}{l}\text { Boro } \\
\text { kalkasunda }\end{array}$ & $\mathrm{H}$ & Rare \\
\hline & $\begin{array}{l}\text { S. siamea (Lam.) H. S. Irwin \& } \\
\text { Barneby }\end{array}$ & Minjiri & $\mathrm{T}$ & Common \\
\hline & S. sophera (L.) Roxb. & Kalkashunda & $\mathrm{S}$ & Common \\
\hline & S. tora (L.) Roxb. & Chakunda & $\mathrm{H}$ & Common \\
\hline & Sesbania bispinosa (Jacq.) Wight. & Dhaincha & $\mathrm{H}$ & Common \\
\hline & Spatholobus parviflorus (DC.) Kuntze & Goalia lata & $\mathrm{Cl}$ & Rare \\
\hline & Tadehagi triquetrum (L.) H. Ohashi & - & $\mathrm{U}$ & Common \\
\hline & Tephrosia candida (Roxb.) DC. & Bilokhoni & $\mathrm{S}$ & Common \\
\hline & T. purpurea (L.) Pers. & Bon neel & $\mathrm{H}$ & Common \\
\hline & Uraria crinita $(\mathrm{L}.) \mathrm{DC}$. & Dieng-kha-riu & $\mathrm{S}$ & Common \\
\hline & U. rufescens (DC.) Schindl. & Belai leza & $\mathrm{S}$ & Common \\
\hline & Vicia sativa $\mathrm{L}$ & Ankari & $\mathrm{H}$ & Common \\
\hline & $\begin{array}{l}\text { Xylia xylocarpa var. kerrii (Craib \& } \\
\text { Hutch.) I.C. Nielsen }\end{array}$ & Lohakat & $\mathrm{T}$ & Rare \\
\hline \multirow[t]{2}{*}{ Polygalaceae } & Salomonia ciliata (L.) DC. & - & $\mathrm{H}$ & Common \\
\hline & Xanthophyllum flavescens Roxb. & Ajensak & $\mathrm{T}$ & Common \\
\hline \multirow[t]{3}{*}{ Rhamnaceae } & Gouania napalensis Wall. & - & $\mathrm{CS}$ & Common \\
\hline & G. tiliifolia Lam. & - & SS & Rare \\
\hline & Ziziphus glabrata Heyne ex Roth. & Pahari boroi & $\mathrm{S}$ & Rare \\
\hline
\end{tabular}


Table 1 Contd.

\begin{tabular}{|c|c|c|c|c|}
\hline Family & Taxa & Bangla name & Habit & $\begin{array}{l}\text { Status of } \\
\text { occurrence }\end{array}$ \\
\hline Rhamnaceae & Z. oenopolia (L.) Mill. & Banboroi & $\mathrm{S}$ & Common \\
\hline Ulmaceae & Trema orientalis (L.) Blume & Jiban & $\mathrm{T}$ & Common \\
\hline \multirow[t]{10}{*}{ Moraceae } & Artocarpus lakoocha Roxb. & Deua & $\mathrm{T}$ & Common \\
\hline & Ficus benjamina $\mathrm{L}$. & Pakur & $\mathrm{T}$ & Common \\
\hline & F. benghalensis L. & Bot & $\mathrm{T}$ & Common \\
\hline & F. fistulosa Reinwdt. ex Blume & - & ST & Common \\
\hline & F. tinctoria subsp. gibbosa (Blume) Corner & Bot & $\mathrm{T}$ & Common \\
\hline & F. heterophylla L. f. & Bhui dumur & $\mathrm{CS}$ & Common \\
\hline & F. hispida L. f. & Kakdumur & ST & Common \\
\hline & F. punctata Thunb. & - & $\mathrm{Cl}$ & Common \\
\hline & F. semicordata Buch.-Ham. ex Sm. & Jagadumur & ST & Common \\
\hline & Streblus asper Lour. & Sheora & $\mathrm{T}$ & Common \\
\hline \multirow[t]{7}{*}{ Urticaceae } & Boehmeria glomerulifera Miq. & Borthurthuri & S & Common \\
\hline & Dendrocnide sinuata (Blume) Chew & - & $\mathrm{S}$ & Common \\
\hline & Oreocnide integrifolia (Gaudich.) Miq. & Horhuta & ST & Common \\
\hline & Pilea microphylla Liebm. & Mariccha lata & $\mathrm{H}$ & Common \\
\hline & Pouzolzia sanguinea (Blume) Merr. & - & $\mathrm{S}$ & Common \\
\hline & P. zeylanica (L.) Benn. & Kullaruki & $\mathrm{H}$ & Common \\
\hline & Sarcochlamys pulcherrima Gaudich & Maricha & $\mathrm{LS}$ & Common \\
\hline Fagaceae & Castanopsis indica (Roxb. ex Lindl.) DC. & Batna & $\mathrm{T}$ & Endangered \\
\hline Betulaceae & Alnus nepalensis G. Don & - & $\mathrm{T}$ & Endangered \\
\hline \multirow[t]{7}{*}{ Cucurbitaceae } & Actinostemma tenerum Griff. & Golapata & $\mathrm{H}$ & Common \\
\hline & Citrullus colocynthis (L.) Schrad. & Indrayan & $\mathrm{Cl}$ & Endangered \\
\hline & Coccinia grandis (L.) Voigt & Telakucha & $\mathrm{H}$ & Common \\
\hline & Hodgsonia macrocarpa (Bl.) Cogn. & Pathligular & $\mathrm{Cl}$ & $\begin{array}{l}\text { Critically } \\
\text { Endangered }\end{array}$ \\
\hline & Thladiantha cordifolia (Bl.) Cogn. & - & $\mathrm{Cl}$ & Rare \\
\hline & Trichosanthes cordata Roxb. & Bhuikakra & $\mathrm{Cl}$ & Rare \\
\hline & T. tricuspidata Lour. & Makal & $\mathrm{H}$ & Rare \\
\hline Datiscaceae & Tetrameles nodiflora $\mathrm{R} . \mathrm{Br}$. & Chundul & $\mathrm{TT}$ & Rare \\
\hline Begoniaceae & Begonia roxburghii (Miq.) A. DC. & - & $\mathrm{H}$ & Endangered \\
\hline \multirow[t]{2}{*}{ Celastraceae } & Euonymus attenuatus Wall. ex Laws. & - & $\mathrm{S}$ & Rare \\
\hline & E. glaber Roxb. & - & ST & Rare \\
\hline Connaraceae & Connarus paniculatus Roxb. & Katgular & WC & Rare \\
\hline \multirow{2}{*}{ Oxalidaceae } & Biophytum sensitivum (L.) DC. & Jhalali & $\mathrm{H}$ & Rare \\
\hline & Oxalis corniculata $\mathrm{L}$ & Amrul & $\mathrm{H}$ & Common \\
\hline Elaeocarpaceae & Elaeocarpus tectorius (Lour.) Poir. & Jalpai & $\mathrm{T}$ & Rare \\
\hline Clusiaceae & Garcinia cowa Roxb. ex Choisy & Kao-gola & MT & Rare \\
\hline Hypericaceae & $\begin{array}{l}\text { Cratoxylum sumatranum subsp. } \\
\text { neriifolium (Kurz) Gogelein }\end{array}$ & Nerikath & LS & Common \\
\hline Achariaceae & Hydnocarpus kurzii (King) Warb. & Chaulmoogra & $\mathrm{T}$ & Rare \\
\hline \multirow[t]{2}{*}{ Passifloraceae } & Adenia trilobata (Roxb.) Engl. & Akandaphal & $\mathrm{Cl}$ & Common \\
\hline & Passiflora foetida $\mathrm{L}$. & Jhumka-lata & $\mathrm{H}$ & Rare \\
\hline Salicaceae & Flacourtia jangomas (Lour.) Racusch. & Paniamala & MT & Endangered \\
\hline \multirow[t]{3}{*}{ Euphorbiaceae } & Acalypha indica $\mathrm{L}$. & Muktajhuri & $\mathrm{H}$ & Common \\
\hline & Astraea lobata (L.) Klotzsch & - & $\mathrm{H}$ & Common \\
\hline & Balakata baccata (Roxb.) Esser & Katagola & $\mathrm{T}$ & Rare \\
\hline
\end{tabular}


Table 1 Contd.

\begin{tabular}{|c|c|c|c|c|}
\hline Family & Taxa & Bangla name & Habit & $\begin{array}{l}\text { Status of } \\
\text { occurrence }\end{array}$ \\
\hline \multirow[t]{21}{*}{ Euphorbiaceae } & $\begin{array}{l}\text { Chaetocarpus castanocarpus (Roxb.) } \\
\text { Thwaites }\end{array}$ & Bul kakra & MT & Common \\
\hline & $\begin{array}{l}\text { Chrozophora rottleri (Geisel.) A. Juss. ex } \\
\text { Spreng. }\end{array}$ & Khudi okra & $\mathrm{H}$ & Common \\
\hline & Cnesmone javanica Blume & Paharibichuti & WC & Common \\
\hline & Croton bonplandianus Baill. & Bondhone & $\mathrm{H}$ & Common \\
\hline & C. caudatus Geisel. & Nanbhanti & $\mathrm{S}$ & Common \\
\hline & Euphorbia hirta L. & Dudhiya & $\mathrm{H}$ & Common \\
\hline & E. thymifolia L. & Swetkan & $\mathrm{H}$ & Rare \\
\hline & Falconeria insignis Royle & Belua & $\mathrm{T}$ & Rare \\
\hline & Jatropha gossypifolia $\mathrm{L}$ & Lal Bherendha & $S$ & Common \\
\hline & Macaranga denticulata (B1.) Müll.Arg. & Bura, Jagra & $\mathrm{T}$ & Common \\
\hline & M. peltata (Roxb.) Müll.Arg. & - & $\mathrm{T}$ & Common \\
\hline & Mallotus nudiflorus (L.) Kulju \& Welzen & Pitali & $\mathrm{T}$ & Rare \\
\hline & M. philippensis (Lam.) Müll.Arg. & Kamela & ST & Rare \\
\hline & M. repandus (Willd.) Müll.Arg. & Gunti & ST & Rare \\
\hline & M. roxburghianus Müll.Arg. & Nim puteli & $\mathrm{S}$ & Rare \\
\hline & M. tetracoccus (Roxb.) Kurz & Kumari-bura & MT & Rare \\
\hline & Manihot esculenta Crantz. & Kasava & $\mathrm{S}$ & Rare \\
\hline & Ricinus communis $\mathrm{L}$. & Verenda & $\mathrm{H}$ & Common \\
\hline & Shirakiopsis indica (Willd.) Esser & Hura & ST & Rare \\
\hline & Suregada multiflora (A. Juss.) Baill. & Maricha & ST & Rare \\
\hline & Tragia involucrata $\mathrm{L}$. & Bichuti & $\mathrm{H}$ & Rare \\
\hline \multirow[t]{22}{*}{ Phyllanthaceae } & Actephila excelsa (Dalzell) Müll.Arg. & & ST & Common \\
\hline & Antidesma acidum Retz. & Chutki & $\mathrm{T}$ & Common \\
\hline & A. bunius (L.) Spreng. & Banshial buka & MT & Rare \\
\hline & A. ghaesembilla Gaertn. & Khudijam & MT & Rare \\
\hline & A. velutinosum $\mathrm{Bl}$. & - & $\mathrm{ST}$ & Rare \\
\hline & Aporosa aurea Hook. f. & Kechuan & $\mathrm{T}$ & Common \\
\hline & $\begin{array}{l}\text { A. octandra (Buch.-Ham. ex D. Don) } \\
\text { Vickery }\end{array}$ & Pat khorulla & ST & Common \\
\hline & A. wallichii Hook.f. & Karullah & $\mathrm{T}$ & Rare \\
\hline & Baccaurea ramiflora Lour. & Latkon & MT & Rare \\
\hline & Bischofia javanica $\mathrm{Bl}$. & Kanjalbhadi & $\mathrm{T}$ & Common \\
\hline & Breynia retusa (Dennst.) Alston & Silpati & $\mathrm{S}$ & Rare \\
\hline & Bridelia stipularis (L.) Bl. & Pat khowi & ST & Common \\
\hline & B. tomentosa $\mathrm{Bl}$. & Khoi & ST & Rare \\
\hline & B. verrucosa Haines & - & ST & Common \\
\hline & Glochidion assamicum (Müll.Arg.) Hook.f. & - & $\mathrm{ST}$ & Rare \\
\hline & G. lanceolarium (Roxb.) Voigt & Bhauri & ST & Common \\
\hline & G. multiloculare (Rottler ex Willd.) Voigt & Keotomi & ST & Common \\
\hline & G. sphaerogynum (Müll.Arg.) Kurz & - & $\mathrm{T}$ & Rare \\
\hline & Phyllanthus attenuatus Miq. & Panjuli & $\mathrm{S}$ & Rare \\
\hline & P. niruri L. & Bhuimala & $\mathrm{H}$ & Common \\
\hline & P. reticulatus Poir. & Chitka & $\mathrm{S}$ & Common \\
\hline & P. sikkimensis Müll.Arg. & Sikim-amla & LS & Common \\
\hline
\end{tabular}


Table 1 Contd.

\begin{tabular}{|c|c|c|c|c|}
\hline Family & Taxa & Bangla name & Habit & $\begin{array}{l}\text { Status of } \\
\text { occurrence }\end{array}$ \\
\hline \multirow[t]{5}{*}{ Combretaceae } & Combretum album Pers. & Kali gumuchi & SS & Rare \\
\hline & C. apetalum Wall. ex Kurz & Dolhara & $\mathrm{S}$ & Rare \\
\hline & Getonia floribunda Roxb. & Guicha lata & SS & Rare \\
\hline & Terminalia alata Heyne ex Roth. & Asal & TT & Rare \\
\hline & T. bellirica (Gaertn.) Roxb. & Bohera & $\mathrm{T}$ & Rare \\
\hline \multirow[t]{4}{*}{ Lythraceae } & Ammannia multiflora Roxb. & - & $\mathrm{H}$ & Common \\
\hline & Lagerstroemia parviflora Roxb. & Baturi & MT & Rare \\
\hline & Woodfordia fruticosa (L.) Kurz & Dhatriphul & $\mathrm{S}$ & Common \\
\hline & $\begin{array}{l}\text { Duabanga grandiflora (Roxb. ex DC.) } \\
\text { Walp. }\end{array}$ & Bandarhulla & $\mathrm{T}$ & Rare \\
\hline \multirow[t]{2}{*}{ Onagraceae } & Ludwigia adscendens (L.) H. Hara & Kesardam & $\mathrm{CH}$ & Common \\
\hline & L. hyssopifolia (G. Don) Exell & - & $\mathrm{H}$ & Common \\
\hline \multirow[t]{2}{*}{ Myrtaceae } & Syzygium nervosum A. Cunn. ex DC. & Bhutijam & $\mathrm{T}$ & Rare \\
\hline & S. fruticosum DC. & Khudijam & $\mathrm{ST}$ & Rare \\
\hline \multirow[t]{2}{*}{ Melastomataceae } & Melastoma malabathricum $\mathrm{L}$. & Bantejpzta & $\mathrm{S}$ & Common \\
\hline & Osbeckia aspericaulis Hook. f. ex Tri. & - & $\mathrm{S}$ & Common \\
\hline Crypteroniaceae & Crypteronia paniculata Blume & Goru-mara & $\mathrm{T}$ & Rare \\
\hline Burseraceae & Protium serratum (Wall. ex Colebr.) Engl. & Gutgutya & $\mathrm{T}$ & Rare \\
\hline \multirow{10}{*}{ Anacardiaceae } & Bouea oppositifolia (Roxb.) Adelb. & Uri Aam & $\mathrm{T}$ & Rare \\
\hline & Buchanania lancifolia Roxb. & Chikki & $\mathrm{T}$ & Rare \\
\hline & $\begin{array}{l}\text { Drimycarpus racemosus (Roxb.) Hook.f. ex } \\
\text { Marchand. }\end{array}$ & Nala-amshi & $\mathrm{T}$ & $\begin{array}{l}\text { Critically } \\
\text { Endangered }\end{array}$ \\
\hline & Holigarna caustica (Dennst.) Oken & Barola & $\mathrm{T}$ & $\begin{array}{l}\text { Critically } \\
\text { Endangered }\end{array}$ \\
\hline & Lannea coromandelica (Houtt.) Merr. & Jiga & $\mathrm{T}$ & Rare \\
\hline & Mangifera laurina $\mathrm{Bl}$. & Jangali aam & $\mathrm{T}$ & Vulnrerable \\
\hline & M. sylvatica Roxb. ex Wall. & Uri Aam & $\mathrm{T}$ & $\begin{array}{l}\text { Critically } \\
\text { Endangered }\end{array}$ \\
\hline & Semecarpus subpanduriformis Wall. & Beula & $\mathrm{T}$ & Rare \\
\hline & Spondias pinnata (L. f.) Kurz & Amra & $\mathrm{T}$ & Rare \\
\hline & Swintonia floribunda Griff. & Chundul & $\mathrm{T}$ & $\begin{array}{l}\text { Critically } \\
\text { Endangered }\end{array}$ \\
\hline \multirow[t]{2}{*}{ Sapindaceae } & Allophylus cobbe (L.) Raeusch. & Chita & $\mathrm{S}$ & Common \\
\hline & A. villosa (Roxb.) Blume & - & ST & Common \\
\hline \multirow[t]{4}{*}{ Sapindaceae } & Cardiospermum halicacabum $\mathrm{L}$. & Phutka & $\mathrm{H}$ & Common \\
\hline & Lepisanthes rubiginosum (Roxb.) Leenh. & Baraharina & ST & Rare \\
\hline & L. senegalensis (Poir.) Leenh. & Gotaharina & $\mathrm{S}$ & Common \\
\hline & Xerospermum laevigatum Radlk. & $\begin{array}{l}\text { Muraillah } \\
\text { lichu }\end{array}$ & $\mathrm{T}$ & Rare \\
\hline \multirow[t]{7}{*}{ Rutaceae } & Aegle marmelos (L.) Corr. & Bel & $\mathrm{T}$ & Rare \\
\hline & Acronychia pedunculata (L.) Miq. & Bon jamir & ST & Rare \\
\hline & Citrus aurantiifolia (Christm.) Sw. & Lebu & $\mathrm{S}$ & Common \\
\hline & Clausena heptaphylla (Roxb.) Wight \& Arn. & Karanphul & $\mathrm{S}$ & Common \\
\hline & Glycosmis pentaphylla (Retz.) DC. & Sheora & $\mathrm{S}$ & Common \\
\hline & Micromelum minutum Wight \& Arn. & Koroiphula & ST & Common \\
\hline & Paramignya scandens (Griff.) Craib. & Bannebu & ST & Rare \\
\hline
\end{tabular}


Table 1 contd.

\begin{tabular}{|c|c|c|c|c|}
\hline Family & Taxa & Bangla name & Habit & $\begin{array}{l}\text { Status of } \\
\text { occurrence }\end{array}$ \\
\hline \multirow[t]{8}{*}{ Meliaceae } & Aglaia edulis (Roxb.) Wall. & & $\mathrm{T}$ & $\begin{array}{l}\text { Critically } \\
\text { endangered }\end{array}$ \\
\hline & Aphanamixis polystachya (Wall.) R. Parker & Pitraj & $\mathrm{T}$ & Common \\
\hline & Azadirachta indica A. Juss. & Neem & $\mathrm{T}$ & Common \\
\hline & Chisocheton paniculatus Hiern & Rata & $\mathrm{T}$ & Rare \\
\hline & Chukrasia tabularis A. Juss. & Chikrassi & $\mathrm{T}$ & Common \\
\hline & Melia azedarach $\mathrm{L}$. & Bokhain & $\mathrm{T}$ & Rare \\
\hline & Swietenia mahagoni (L.) Jacq. & Mehogini & $\mathrm{T}$ & Common \\
\hline & Toona ciliata $\mathrm{M}$. Roem & Toon & $\mathrm{T}$ & Common \\
\hline \multirow[t]{28}{*}{ Malvaceae } & Abelmoschus moschatus Medik. & Mushakdana & $\mathrm{H}$ & Common \\
\hline & Abroma augusta (L.) L. f. & Ulatkambal & ST & Common \\
\hline & Abutilon indicum (L.) Sweet & Petari & $\mathrm{H}$ & Common \\
\hline & Bombax ceiba $\mathrm{L}$. & Shimul & $\mathrm{T}$ & Common \\
\hline & B. insigne Wall. & Ban-simul & $\mathrm{T}$ & Rare \\
\hline & Brownlowia elata Roxb. & Masjot & $\mathrm{T}$ & $\begin{array}{l}\text { Critically } \\
\text { endangered }\end{array}$ \\
\hline & Byttneria pilosa Roxb. & Harjora-lata & WC & Common \\
\hline & Ceiba pantandra (L.) Gaertn. & Pahari tula & $\mathrm{T}$ & Rare \\
\hline & Corchorus aestuans L. & Titapat & $\mathrm{H}$ & Rare \\
\hline & Firmiana colorata (Roxb.) R. Br. & Patagota & $\mathrm{T}$ & $\begin{array}{l}\text { Critically } \\
\text { endangered }\end{array}$ \\
\hline & Grewia asiatica $\mathrm{L}$. & Pholsa & ST & Common \\
\hline & G. laevigata Vahl & Panisara & ST & Common \\
\hline & G. nervosa (Lour.) Panigrahi & Asar & ST & Common \\
\hline & G. serrulata DC. & Panisara & $\mathrm{T}$ & Rare \\
\hline & Hibiscus vitifolius L. & Bon-karpas & $\mathrm{H}$ & Common \\
\hline & Malachra capitata $\mathrm{L}$. & Bon vindi & $\mathrm{H}$ & Rare \\
\hline & Pterospermum acerifolium (L.)Willd. & Musigondha & $\mathrm{T}$ & Endangered \\
\hline & P. semisagittatum Buch.-Ham. ex Roxb. & Bara assar & $\mathrm{T}$ & Endangered \\
\hline & Sida acuta Burm. f. & Kureta & $\mathrm{H}$ & Common \\
\hline & S. cordata (Burm. f.) Borss. Waalk. & Jhumka & $\mathrm{H}$ & Common \\
\hline & S. cordifolia $\mathrm{L}$ & Berela & $\mathrm{H}$ & Common \\
\hline & S. mysorensis Wight \& Arn. & Chatehata & $\mathrm{H}$ & Common \\
\hline & Sterculia foetida $\mathrm{L}$. & Udal & MT & Endangered \\
\hline & S. hamiltonii (Kuntze) Adelb. & - & $\mathrm{ST}$ & Endangered \\
\hline & S. villosa Roxb. ex Smith & Udal & MT & Endangered \\
\hline & Triumfetta pilosa Roth & - & $\mathrm{H}$ & Common \\
\hline & T. rhomboidea Jacq. & Ban okra & $\mathrm{H}$ & Common \\
\hline & Urena lobata $\mathrm{L}$. & Banokra & $\mathrm{H}$ & Common \\
\hline Bixaceae & Bixa orellana $\mathrm{L}$ & Latkon & ST & Rare \\
\hline \multirow[t]{4}{*}{ Dipterocarpaceae } & Anisoptera scaphula (Roxb.) Pierre. & Boilsur & $\mathrm{T}$ & $\begin{array}{l}\text { Critically } \\
\text { endangered }\end{array}$ \\
\hline & Dipterocarpus costatus & Garjan & $\mathrm{T}$ & Rare \\
\hline & D. turbinatus Gaertn. & Teli garjan & $\mathrm{T}$ & Common \\
\hline & Hopea odorata Roxb. & Telsur & $\mathrm{T}$ & Common \\
\hline \multirow[t]{3}{*}{ Capparaceae } & Capparis spinosa $\mathrm{L}$. & Kabra & $\mathrm{S}$ & Rare \\
\hline & Cleome rutidosperma $\mathrm{DC}$. & Begunehurhury & $\mathrm{H}$ & Common \\
\hline & C. viscosa $\mathrm{L}$. & Holdehurhury & $\mathrm{H}$ & Common \\
\hline
\end{tabular}


Table 1 Contd.

\begin{tabular}{|c|c|c|c|c|}
\hline Family & Taxa & Bangla name & Habit & $\begin{array}{l}\text { Status of } \\
\text { occurrence }\end{array}$ \\
\hline \multirow[t]{2}{*}{ Capparaceae } & Crateva religiosa $\mathrm{G}$. Forst. & Barun & ST & Common \\
\hline & Capsella bursa-pastoris (L.) Medik. & Capsala & $\mathrm{H}$ & Common \\
\hline \multirow[t]{2}{*}{ Brassicaceae } & Rorippa indica (L.) Hiern & Ban-saruisha & $\mathrm{H}$ & Common \\
\hline & R. palustris (L.) Besser & Panisarisha & $\mathrm{H}$ & Common \\
\hline \multirow[t]{4}{*}{ Loranthaceae } & Dendrophthoe pentandra (L.) Miq. & - & $\mathrm{S}$ & Rare \\
\hline & Macrosolen cochinchinensis (Lour.) Van Tiegh. & Chota banda & $\mathrm{S}$ & Rare \\
\hline & Scurrula gracilifolia (Schult.) Danser & Pargacha & $\mathrm{S}$ & Rare \\
\hline & S. parasitica $\mathrm{L}$. & Parula & $\mathrm{S}$ & Rare \\
\hline \multirow[t]{5}{*}{ Polygonaceae } & Persicaria chinensis (L.) H.Gross & $\begin{array}{l}\text { Mohicharan } \\
\text { sak }\end{array}$ & $\mathrm{H}$ & Common \\
\hline & P. hydropiper (L.) Delarbre & Bishkatali & $\mathrm{H}$ & Common \\
\hline & $P$. orientalis $(\mathrm{L}$.$) Spach$ & Panimarich & $\mathrm{H}$ & Common \\
\hline & Polygonum viscosum Buch.-Ham. ex D. Don & $\begin{array}{l}\text { Athalo } \\
\text { bishkatali }\end{array}$ & $\mathrm{H}$ & Rare \\
\hline & Rumex vesicarius L. & Tokpalong & $\mathrm{H}$ & Rare \\
\hline \multirow[t]{9}{*}{ Amaranthaceae } & Achyranthes aspera $\mathrm{L}$. & Apang & $\mathrm{H}$ & Common \\
\hline & Alternanthera philoxeroides (Mart.) Griseb. & Sachishak & $\mathrm{H}$ & Common \\
\hline & A. sessilis (L.) R. Br. ex DC. & Chanchi & $\mathrm{H}$ & Common \\
\hline & Amaranthus spinosus L. & Kantanotey & $\mathrm{H}$ & Common \\
\hline & A. viridis L. & Notey shak & $\mathrm{H}$ & Common \\
\hline & Celosia argentea $\mathrm{L}$. & $\begin{array}{l}\text { Shet } \\
\text { morogha }\end{array}$ & $\mathrm{H}$ & Common \\
\hline & Chenopodium album $\mathrm{L}$. & Batua Shak & $\mathrm{S}$ & Common \\
\hline & Cyathula prostrata (L.) Blume & Shyontula & $\mathrm{H}$ & Common \\
\hline & Gomphrena celosioides Mart. & - & $\mathrm{H}$ & Common \\
\hline Nyctaginaceae & Boerhavia repens $\mathrm{L}$. & Punarnava & $\mathrm{H}$ & Common \\
\hline \multirow[t]{2}{*}{ Molluginaceae } & Glinus oppositifolius (L.) A. DC. & Gimashakh & $\mathrm{H}$ & Common \\
\hline & Mollugo stricta $\mathrm{L}$. & Khetpapra & $\mathrm{H}$ & Rare \\
\hline Portulacaceae & Portulaca oleracea $\mathrm{L}$. & Nune shak & $\mathrm{H}$ & Common \\
\hline Lecythidaceae & Barringtonia acutangula (L.) Gaertn. & Hijal & $\mathrm{T}$ & Common \\
\hline \multirow{2}{*}{ Ebenaceae } & Diospyros malabarica (Desr.) Kostl. & Deshi gab & $\mathrm{T}$ & Rare \\
\hline & D. racemosa Roxb. & $\mathrm{Gab}$ & MT & Endangered \\
\hline \multirow[t]{3}{*}{ Primulaceae } & Ardisia humilis Vahl & Chauldhoa & $\mathrm{S}$ & Common \\
\hline & A. paniculata Roxb. & - & ST & Rare \\
\hline & A. sanguinolenta Blume & - & ST & Endangered \\
\hline \multirow[t]{3}{*}{ Primulaceae } & A. solanacea (Poir.) Roxb. & Bon -jam & $\mathrm{S}$ & Common \\
\hline & Maesa indica (Roxb.) A. DC. & Sirkhi & $\mathrm{S}$ & Common \\
\hline & M. ramentacea (Roxb.) A. DC. & Naricha & $\mathrm{ST}$ & Common \\
\hline Actinidiaceae & Saurauia roxburghii Wall. & Bhola kadam & $\mathrm{S}$ & Rare \\
\hline \multirow[t]{8}{*}{ Rubiaceae } & Canthium angustifolium Roxb. & Katamalli & S & Common \\
\hline & Ceriscoides campanulata (Roxb.) Tirveng. & Tirveng & $\mathrm{T}$ & Endangered \\
\hline & $\begin{array}{l}\text { Chassalia curviflora var. ophioxyloides (Wall.) } \\
\text { Deb \& B.Krishna }\end{array}$ & - & S & Rare \\
\hline & Dentella repens (L.) J.R. Forst. \& G. Frost. & Bhuipat & $\mathrm{H}$ & Common \\
\hline & Gardenia coronaria Buch.-Ham. & Torgular & MT & Rare \\
\hline & Haldina cordifolia (Roxb.) Ridsd. & Kalakadam & $\mathrm{T}$ & Rare \\
\hline & Hedyotis scandens Roxb. & Bish lata & $\mathrm{H}$ & Common \\
\hline & $\begin{array}{l}\text { Hyptianthera stricta (Roxb. ex Schult.) Wight \& } \\
\text { Arn. }\end{array}$ & - & $\mathrm{S}$ & Common \\
\hline
\end{tabular}


Table 1 Contd.

\begin{tabular}{|c|c|c|c|c|}
\hline Family & Taxa & Bangla name & Habit & $\begin{array}{l}\text { Status of } \\
\text { occurrence }\end{array}$ \\
\hline \multirow[t]{39}{*}{ Rubiaceae } & Ixora athrorantha Bremek. & - & ST & Common \\
\hline & I. balakrishnanii Deb \& Rout & Bhantjhara & ST & Common \\
\hline & I. cuneifolia Roxb. & Beophul & $\mathrm{S}$ & Common \\
\hline & I. nigricans $\mathrm{R} . \mathrm{Br}$. ex Wight \& Arn. & Nikranga & $\mathrm{ST}$ & Common \\
\hline & I. pavetta Andr. & Swetrangan & ST & Common \\
\hline & I. pubirama Bremek. & Keamosi & $\mathrm{S}$ & Common \\
\hline & I. spectabilis Wall. ex G. Don & - & ST & Common \\
\hline & I. subsessilis Wall. ex G. Don & Rengchan & $\mathrm{S}$ & Common \\
\hline & Knoxia sumatrensis (Retz.) DC. & - & $\mathrm{H}$ & Common \\
\hline & Lasianthus hirsutus (Roxb.) Merr. & Kala long & $\mathrm{S}$ & Common \\
\hline & Meyna spinosa Roxb. ex Link & Mainakata & ST & Rare \\
\hline & Mitracarpus hirtus (L.) DC. & - & $\mathrm{H}$ & Common \\
\hline & $\begin{array}{l}\text { Mitragyna diversifolia (Wall. ex G. Don) } \\
\text { Havil. }\end{array}$ & Phul-kadam & $\mathrm{T}$ & Common \\
\hline & $\begin{array}{l}\text { M. parvifolia var. microphylla (Kurz) } \\
\text { Ridsdale }\end{array}$ & Putikadam & $\mathrm{T}$ & Near threatened \\
\hline & Morinda angustifolia Roxb. & Banamali & ST & Common \\
\hline & M. citrifolia $\mathrm{L}$. & Ronch & ST & Common \\
\hline & M. persicifolia Buch.-Ham. & - & $\mathrm{S}$ & Common \\
\hline & Mussaenda roxburghii Hook. f. & Sildaura & S & Common \\
\hline & Neolamarckia cadamba (Roxb.) Boss. & Kadom & $\mathrm{T}$ & Common \\
\hline & Neonauclea sessilifolia (Roxb.) Merr. & Kom & $\mathrm{T}$ & Near threatened \\
\hline & Oldenlandia auricularia (L.) K. Sch. & Mutia lata & $\mathrm{H}$ & Common \\
\hline & O. corymbosa $\mathrm{L}$. & Ketpapra & $\mathrm{H}$ & Common \\
\hline & O. diffusa (Willd.) Roxb. & - & $\mathrm{H}$ & Common \\
\hline & Ophiorrhiza mungos $\mathrm{L}$. & Kalashana & $\mathrm{H}$ & Common \\
\hline & $\begin{array}{l}\text { O. rugosa var. prostrata (D. Don) Deb \& } \\
\text { Mondal }\end{array}$ & Jari & $\mathrm{H}$ & Common \\
\hline & $\begin{array}{l}\text { Oxyceros kunstleri (King \& Gamble) } \\
\text { Tirveng. }\end{array}$ & Maish kata & CS & Rare \\
\hline & Paederia foetida $\mathrm{L}$ & Gandha-badali & $\mathrm{Cl}$ & Common \\
\hline & Pavetta indica $\mathrm{L}$ & Banamali & ST & Common \\
\hline & $\begin{array}{l}\text { Prismatomeris tetrandra (Roxb.) K. } \\
\text { Schum. }\end{array}$ & Chinatita & ST & Common \\
\hline & Psychotria adenophylla Wall. & Baro sudma & ST & Common \\
\hline & P. calocarpa Kurz & Ranga bhutta & $\mathrm{U}$ & Common \\
\hline & P. monticola Kurz & Hatichotra & $\mathrm{S}$ & Common \\
\hline & P. symplocifolia Kurz & - & $\mathrm{ST}$ & Common \\
\hline & Spermacoce articularis L.f. & Ahtharogia & $\mathrm{H}$ & Common \\
\hline & S. hispida $\mathrm{L}$. & Ahtharogia & $\mathrm{H}$ & Common \\
\hline & $\begin{array}{l}\text { Tarenna companiflora (Hook. f.) N.P. } \\
\text { Balakr. }\end{array}$ & Kakra & ST & Common \\
\hline & Tarennoidea wallichii Tirveng. \& Sast. & - & $\mathrm{T}$ & Common \\
\hline & Uncaria scandens (Smith) Hutch. & Vailful lata & $\mathrm{Cl}$ & Rare \\
\hline & $\begin{array}{l}\text { Wendlandia tinctoria subsp. orientalis } \\
\text { Cowan }\end{array}$ & Tulaload & ST & Common \\
\hline \multirow[t]{2}{*}{ Gentianaceae } & Exacum tetragonum Roxb. & Kuchuri & $\mathrm{H}$ & Rare \\
\hline & Fagraea ceilanica Thunb. & - & ST & Rare \\
\hline
\end{tabular}


Table 1 Contd.

\begin{tabular}{|c|c|c|c|c|}
\hline Family & Taxa & Bangla name & Habit & $\begin{array}{l}\text { Status of } \\
\text { occurrence }\end{array}$ \\
\hline \multirow[t]{18}{*}{ Apocynaceae } & Alstonia scholaris (L.) R. Br. & Chaitan & $\mathrm{T}$ & Common \\
\hline & Anodendron paniculatum A.DC. & Dul & WC & Rare \\
\hline & Asclepias curassavica $\mathrm{L}$. & Moricha & $\mathrm{H}$ & Rare \\
\hline & Calotropis gigantea (L.) Dryand. & Akanda & $\mathrm{S}$ & Common \\
\hline & Chonemorpha fragrans (Moon) Alston & Gar badero & WC & Endangered \\
\hline & Gymnema acuminatum Wall. & Khara lata & WT & Rare \\
\hline & Hemidesmus indicus (L.) R.Br. ex Schult. & Anantamul & $\mathrm{H}$ & Rare \\
\hline & $\begin{array}{l}\text { Holarrhena pubescens (Roth) Wall. ex A. } \\
\text { DC. }\end{array}$ & Kurchi, Kuruj & ST & Rare \\
\hline & Hoya globulosa Hook. f. & Pargacha & Ep & Rare \\
\hline & H. parasitica (Roxb.) Wall. ex Wight & Pargacha & $\mathrm{E}$ & Common \\
\hline & Ichnocarpus frutescens (L.) W. T. Aiton & Syamalota & $\mathrm{CS}$ & Rare \\
\hline & Melodinus cochinchinensis (Lour.) Merr. & - & WT & Vulnerable \\
\hline & Rauvolfia serpentina (L.) Benth. ex Kurz & Sarpagandha & $\mathrm{H}$ & Endangered \\
\hline & Strophanthus wallichii A. DC. & - & WC & Endangered \\
\hline & $\begin{array}{l}\text { Tabernaemontana divaricata (L.) R. Br. } \\
\text { ex Roem. \& Schult. }\end{array}$ & Togor & $\mathrm{S}$ & Common \\
\hline & Tylophora hirsuta Wight & Anantamul & Tw & Rare \\
\hline & Vallaris solanacea (Roth) Kuntze & Hadpur & SS & $\begin{array}{l}\text { Critically } \\
\text { endangered }\end{array}$ \\
\hline & Wrightia arborea (Dennst.) Mabb. & Dudhkurush & $\mathrm{T}$ & Endangered \\
\hline \multirow[t]{2}{*}{ Boraginaceae } & Cordia dichotoma $\mathrm{G}$. Forst. & Buha & $\mathrm{T}$ & Rare \\
\hline & Heliotropium indicum $\mathrm{L}$. & Hatisur & $\mathrm{H}$ & Common \\
\hline \multirow[t]{20}{*}{ Convolvulaceae } & Argyreia argentea (Roxb.) Choisy. & Bara rupatola & $\mathrm{Cl}$ & Common \\
\hline & A. capitiformis (Poir.) Ooststr. & Vogalata & $\mathrm{Cl}$ & Rare \\
\hline & A. splendens (Hornem.) Sweet & Borogandobaduli & $\mathrm{Cl}$ & Rare \\
\hline & Calystegia hederacea Wall. & - & Tw & Common \\
\hline & Cuscuta reflexa Roxb. & Swornalata & $\mathrm{P}$ & Rare \\
\hline & Evolvulus nummularius (L.) L. & Bhui-akra & $\mathrm{H}$ & Common \\
\hline & Hewittia malabarica (L.) Suresh & - & $\mathrm{Cl}$ & Common \\
\hline & Ipomoea alba $\mathrm{L}$ & Dudhikalmi & Tw & Rare \\
\hline & $\begin{array}{l}\text { I. aculeata var. mollissima (Zoll.) Hallier } \\
\text { f. ex Oostr. }\end{array}$ & - & $\mathrm{Cl}$ & Rare \\
\hline & I. aquatica Forssk. & Kalmishak & $\mathrm{AH}$ & Common \\
\hline & I. carnea Jacq. & Dholkolmi & $\mathrm{H}$ & Common \\
\hline & I. fistulosa Mart. ex Choisy & Dhol Kolmi & $\mathrm{S}$ & Common \\
\hline & I. hederifolia $\mathrm{L}$. & Neela-kalmi & $\mathrm{Tw}$ & Rare \\
\hline & I. mauritiana Jacq. & Bhui-Kumra & $\mathrm{Tw}$ & Rare \\
\hline & I. pes-tigridis L. & Languli lata & $\mathrm{Cl}$ & Common \\
\hline & I. pileata Roxb. & - & $\mathrm{Cl}$ & Common \\
\hline & $\begin{array}{l}\text { Jacquemontia paniculata (Burm. f.) } \\
\text { Hallier f. }\end{array}$ & Montilata & $\mathrm{Tw}$ & Rare \\
\hline & Merremia umbellata (L.) Hallier & Sadakamni & $\mathrm{Cl}$ & Common \\
\hline & M. vitifolia (Burm. f.) Hallier f. & - & Tw & Common \\
\hline & Operculina turpethum (L.) Silva Manso & Dudh kalmi & Tw & Rare \\
\hline
\end{tabular}


Table 1 Contd.

\begin{tabular}{|c|c|c|c|c|}
\hline Family & Taxa & Bangla name & Habit & $\begin{array}{l}\text { Status of } \\
\text { occurrence }\end{array}$ \\
\hline \multirow[t]{11}{*}{ Solanaceae } & Datura metel L. & Kala datura & $\mathrm{H}$ & Common \\
\hline & Nicotiana plumbaginifolia Viv. & Bon tamak & $\mathrm{H}$ & Common \\
\hline & Physalis angulata $\mathrm{L}$. & Fotka & $\mathrm{H}$ & Common \\
\hline & P. micrantha Link. & Bantipariya & $\mathrm{H}$ & Common \\
\hline & P. minima $\mathrm{L}$. & Phutka & $\mathrm{H}$ & Common \\
\hline & Solanum ilicifolium Dunal & Tepari & $\mathrm{H}$ & Common \\
\hline & S. indicum L. & Bon Begun & $\mathrm{S}$ & Common \\
\hline & S. nigrum L. & Kakmachi & $\mathrm{H}$ & Common \\
\hline & S. sisymbrifolium Lam. & Kanta begun & $\mathrm{H}$ & Common \\
\hline & S. torvum Sw. & Tit begun & $\mathrm{S}$ & Common \\
\hline & S. virginianum L. & Kantakari & $\mathrm{H}$ & Common \\
\hline \multirow[t]{3}{*}{ Oleaceae } & Jasminum scandens Vahl & - & WC & Rare \\
\hline & Ligustrum robustum (Roxb.) Blume & - & LS & Rare \\
\hline & Myxopyrum smilacifolium (Wall.) Bl. & - & SS & Rare \\
\hline \multirow[t]{8}{*}{ Scrophulariaceae } & Adenosma indianum (Lour.) Merr. & Barakesuti & $\mathrm{H}$ & Common \\
\hline & Buddleja asiatica Lour. & Neemda & $\mathrm{S}$ & Rare \\
\hline & Lindernia antipoda (L.) Alston & - & $\mathrm{H}$ & Common \\
\hline & Picria fel-terrae Lour. & - & $\mathrm{H}$ & Rare \\
\hline & Scoparia dulcis L. & Bandhane & $\mathrm{H}$ & Common \\
\hline & Torenia asiatica $\mathrm{L}$. & - & $\mathrm{H}$ & Common \\
\hline & T. diffusa D. Don & - & $\mathrm{H}$ & Common \\
\hline & T. flava Buch.-Ham. ex Benth. & - & $\mathrm{H}$ & Common \\
\hline \multirow[t]{22}{*}{ Acanthaceae } & Andrographis laxiflora (B1.) Lindau & Algatita & $\mathrm{H}$ & Rare \\
\hline & A. paniculata (Burm. f.) Nees & Kalmegh & $\mathrm{H}$ & Rare \\
\hline & Barleria strigosa Willd. & Katapol & $\mathrm{H}$ & Rare \\
\hline & Dicliptera chinensis (L.) Juss. & - & $\mathrm{H}$ & Rare \\
\hline & Ecbolium ligustrinum (Vahl) Voll. & - & $\mathrm{S}$ & Rare \\
\hline & Eranthemum pulchellum Andrew & - & $\mathrm{S}$ & Common \\
\hline & E. strictum Colebr. ex Roxb. & Khara murali & $\mathrm{H}$ & Rare \\
\hline & Justicia adhatoda $\mathrm{L}$. & Basak & $\mathrm{S}$ & Common \\
\hline & J. gendarussa Burm. f. & Jugmadan & $\mathrm{U}$ & Common \\
\hline & Hygrophila polysperma (Roxb.) T. Anders & - & $\mathrm{H}$ & Common \\
\hline & H. ringens (L.) R. Br. ex Spreng. & Kakmasha & $\mathrm{H}$ & Common \\
\hline & Lepidagathis incurva Buch.-Ham. ex D. Don & Karoggthis & $\mathrm{H}$ & Common \\
\hline & Nelsonia canescens (Lam.) Spreng. & Paramul & $\mathrm{H}$ & Common \\
\hline & Phaulopsis imbricata (Forssk.) Sweet & Kantasi & $\mathrm{H}$ & Common \\
\hline & Ruellia acuminata $\mathrm{L}$. & - & $\mathrm{H}$ & Common \\
\hline & R. tuberosa L. & Chotpoty & $\mathrm{H}$ & Common \\
\hline & Rungia pectinata (L.) Nees & Pindi & $\mathrm{H}$ & Common \\
\hline & Staurogyne argentea Wall. & - & $\mathrm{H}$ & Rare \\
\hline & Strobilanthes auriculatus Nees & Kurinji & $\mathrm{S}$ & Rare \\
\hline & S. scaber Nees & - & $\mathrm{H}$ & Rare \\
\hline & Thunbergia alata Bojer ex Sims & Ghontolata & $\mathrm{Cl}$ & Rare \\
\hline & T. grandiflora (Roxb. ex Rottl.) Roxb. & Nallata & $\mathrm{Cl}$ & Common \\
\hline \multirow[t]{3}{*}{ Bignoniaceae } & Oroxylum indicum (L.) Kurz & Thona & $\mathrm{T}$ & Rare \\
\hline & Stereospermum chelonoides (L.f.) DC. & Kam sonalu & $\mathrm{T}$ & Rare \\
\hline & S. tetragonum DC. & Dharmara & $\mathrm{T}$ & Rare \\
\hline
\end{tabular}


Table 1 Contd.

\begin{tabular}{|c|c|c|c|c|}
\hline Family & Taxa & Bangla name & Habit & $\begin{array}{l}\text { Status of } \\
\text { occurrence }\end{array}$ \\
\hline \multirow[t]{4}{*}{ Verbenaceae } & Duranta erecta $\mathrm{L}$. & Kantamehedi & SS & Common \\
\hline & Lantana camara $\mathrm{L}$ & Lantana & $\mathrm{S}$ & Common \\
\hline & $\begin{array}{l}\text { Lippia alba (Mill.) N.E. Br. ex Britton \& P. } \\
\text { Wilson }\end{array}$ & Motmotia & $\mathrm{S}$ & Common \\
\hline & Phyla nodiflora (L.) Greene & Bhuiokra & $\mathrm{H}$ & Common \\
\hline \multirow[t]{21}{*}{ Lamiaceae } & Anisomeles indica (L.) O. Kuntze & Gobura & $\mathrm{H}$ & Common \\
\hline & Callicarpa arborea Roxb. & Bormala & $\mathrm{T}$ & Rare \\
\hline & C. macrophylla Vahl & Bormala & $\mathrm{T}$ & Rare \\
\hline & Clerodendrum indicum (L.) Kuntze & Bamunhatti & $\mathrm{H}$ & Common \\
\hline & C. infortunatum $\mathrm{L}$. & Ghetu & $\mathrm{S}$ & Common \\
\hline & C. laevifolium $\mathrm{Bl}$. & - & $\mathrm{S}$ & Common \\
\hline & Gmelina arborea Roxb. & Gamar & $\mathrm{H}$ & Common \\
\hline & Hyptis brevipes Poit. & - & $\mathrm{H}$ & Common \\
\hline & H. capitata Jacq. & - & $\mathrm{H}$ & Common \\
\hline & H. suaveolens (L.) Poit. & Tokma & $\mathrm{H}$ & Common \\
\hline & Leonurus sibiricus L. & Roktodron & $\mathrm{H}$ & Rare \\
\hline & Leucas aspera (Willd.) Link & Shetodron & $\mathrm{H}$ & Common \\
\hline & L. zeylanica (L.) Aiton & Dondokolosh & $\mathrm{H}$ & Common \\
\hline & Ocimum americanum $\mathrm{L}$. & Bon Tulsi & $\mathrm{H}$ & Common \\
\hline & O. basilicum L. & Babuitulshi & $\mathrm{H}$ & Common \\
\hline & Pogostemon auricularius (L.) Hassk. & - & $\mathrm{H}$ & Common \\
\hline & Premna esculenta Roxb. & Lalana & $\mathrm{S}$ & Common \\
\hline & Vitex altissima L.f. & Awalya & $\mathrm{T}$ & Rare \\
\hline & V. glabrata $\mathrm{R} . \mathrm{Br}$. & Ashal & $\mathrm{T}$ & Rare \\
\hline & V. negundo $\mathrm{L}$. & Nishinda & ST & Common \\
\hline & V. peduncularis Wall. ex Schauer & Awal & ST & Rare \\
\hline Campanulaceae & Cyclocodon lancifolius (Roxb.) Kurz & - & $\mathrm{H}$ & Rare \\
\hline \multirow[t]{19}{*}{ Asteraceae } & Acmella paniculata (Wall. ex DC.) R.K. Jansen & Kannaghas & $\mathrm{H}$ & Common \\
\hline & Adenostemma lavenia (L.) Kuntze & Baro-kesuti & $\mathrm{H}$ & Common \\
\hline & A. viscosum J. R. Forst. \& G. Forst. & Boro kesuti & $\mathrm{H}$ & Rare \\
\hline & Ageratum conyzoides (L.) L. & Fulkuri & $\mathrm{H}$ & Common \\
\hline & Blumea fistulosa (Roxb.) Kurz & - & $\mathrm{H}$ & Common \\
\hline & B. lacera (Burm. f.) DC. & Kuksung & $\mathrm{H}$ & Common \\
\hline & B. lanceolaria (Roxb.) Druce & - & $\mathrm{H}$ & Common \\
\hline & B. virens DC. & Nilsabus & $\mathrm{H}$ & Common \\
\hline & Blumeopsis flava (DC.) Gagnep & - & $\mathrm{H}$ & Common \\
\hline & Chromolaena odorata (L.) R. M. King \& H. Rob. & Assam-lata & $\mathrm{H}$ & Common \\
\hline & Crassocephalum crepidioides S. Moore & - & $\mathrm{H}$ & Common \\
\hline & Cyanthillium cinereum (L.) H. Rob. & Sahadebi & $\mathrm{H}$ & Common \\
\hline & Eclipta prostrata $(\mathrm{L}.) \mathrm{L}$. & Kesraj & $\mathrm{H}$ & Common \\
\hline & Elephantopus scaber L. & Gejiashak & $\mathrm{H}$ & Common \\
\hline & Emilia sonchifolia (L.) DC. ex DC. & Mechitra & $\mathrm{H}$ & Common \\
\hline & Grangea maderaspatana (L.) Poir. & Namuti & $\mathrm{H}$ & Rare \\
\hline & Laphangium luteoalbum (L.) Tzvelev & Dudh ghas & $\mathrm{H}$ & Common \\
\hline & Mikania scandens (L.) Willd. & Tufanilata & $\mathrm{H}$ & Common \\
\hline & $\begin{array}{l}\text { Pseudelephantopus spicatus (B.Juss. ex Aubl.) } \\
\text { Rohr ex Baker }\end{array}$ & Kukurgihba & $\mathrm{H}$ & Rare \\
\hline
\end{tabular}


Table 1 Contd.

\begin{tabular}{|c|c|c|c|c|}
\hline Family & Taxa & Bangla name & Habit & $\begin{array}{l}\text { Status of } \\
\text { occurrence }\end{array}$ \\
\hline \multirow[t]{8}{*}{ Asteraceae } & Sonchus wightianus DC. & - & $\mathrm{H}$ & Common \\
\hline & Sphaeranthus africanus L. & Gongasag & $\mathrm{H}$ & Rare \\
\hline & S. indicus L. & Murmuri & $\mathrm{H}$ & Rare \\
\hline & S. senegalensis DC. & Senigalsag & $\mathrm{H}$ & Rare \\
\hline & Spilanthes acmella (L.) L. & Nag phul & $\mathrm{H}$ & Common \\
\hline & Synedrella nodiflora (L.) Gaertn. & Relanodi & $\mathrm{H}$ & Common \\
\hline & Tridax procumbens $\mathrm{L}$. & Tridhara & $\mathrm{H}$ & Common \\
\hline & Xanthium strumarium L. & Ghagra & $\mathrm{H}$ & Common \\
\hline \multirow[t]{2}{*}{ Araliaceae } & Brassaiopsis glomerulata (B1.) Regel & Kurila & ST & Common \\
\hline & Trevesia palmata (Roxb. ex Lindl.)Vis. & Vombal & LS & Common \\
\hline \multirow[t]{2}{*}{ Apiaceae } & Centella asiatica (L.) Urb. & Thankuni & $\mathrm{H}$ & Common \\
\hline & Hydrocotyle sibthorpioides Lam. & - & $\mathrm{H}$ & Common \\
\hline
\end{tabular}

$\mathrm{H}=$ Herb, $\mathrm{CS}=$ Climbing shrub, $\mathrm{Cr}=$ Creeper, WT $=$ Woody twinner, $\mathrm{T}=$ Tree $, \mathrm{S}=\mathrm{Shrub}, \mathrm{Cl}=\mathrm{Cimber}$, WC= Woody climber, TS= Twining shrub, $\mathrm{SS}=\mathrm{Scandent}$ shrub, Tw= Twiner, $\mathrm{U}=$ undershrub, $\mathrm{LS}=\mathrm{Large}$ shrub, $\mathrm{MT}=$ Medium-sized tree, $\mathrm{ST}=$ Small tree, $\mathrm{CH}=$ Creeping herb, $\mathrm{E}=$ Epiphyte, $\mathrm{P}=$ Parasite, $\mathrm{AH}=$ Aquatic herb.

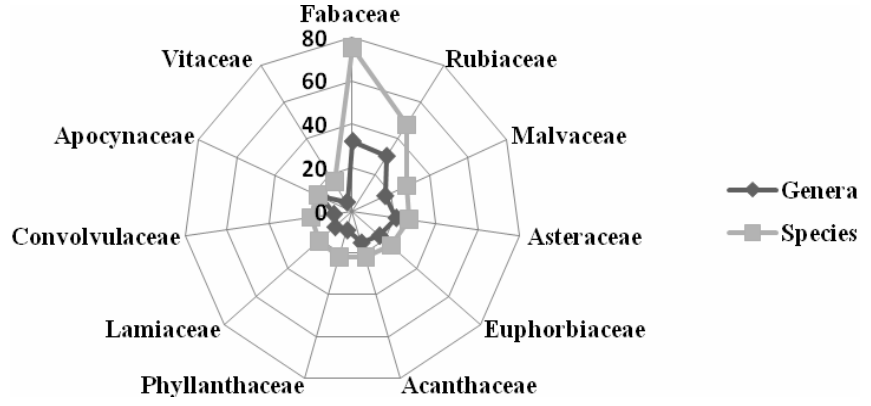

Fig. 2. Radar diagram shows ten dominat plant families in the Baraiyadhala National Park.

critically endangered and 4 species $(0.76 \%)$ as vulnerable. All species belonging to the families Anacardiaceae (10 spp.), Bignoniaceae (3 spp.), Dipterocarpaceae (4 spp.), and Meliaceae (8 spp.) are trees and under threat in the forest. Rahman (2013) reported Artabotrys caudatus of the family Annonaceae as possibly extinct in Bangladesh. Recently this species has been reported from Hazarikhil Wildlife Sanctuary by Rahman (2017) and current investigation also reports its distribution in the Baraiyadhala National Park.

The present study reveals that communities around the area depend more or less on forest resources and some organized encroachers are also noticed. Furthermore, forest fire, livestock and other unsustainable resource utilization practices degrade the habitat in and around the forest. Climate change is an additional threat which may directly affect habitat and biodiversity, as well as indirectly by further increasing human pressure due to migration of peoples from coastal parts of Sitakunda and Mirsharai to the forest area. Development of tourism may enhance a future threat to the park. To address these challenges sustainable management plan for the protected area should be undertaken in the light of National Conservation Strategy, and proper implementation of the action plan is urgently required with collaboration and cooperation of stakeholders and local administrations. More particularly, the rare and threatened species along with their degraded habitats to be protected by taking proper conservation management programmes. 


\section{Acknowledgements}

This study was supported by Research Allocation Fund under Revenue Budget, University of Chittagong, Bangladesh. We are thankful to Prof. Dr. M. Atiqur Rahman, Department of Botany, University of Chittagong for his generous help and cooperation during the course of this study.

\section{References}

Ahmed, Z.U., Begum, Z.N.T., Hassan, M.A., Khondker, M., Kabir, S.M.H., Ahmad, M., Ahmed, A.T.A., Rahman, A.K.A. and Haque, E.U. (Eds) 2008. Encyclopedia of Flora and Fauna of Bangladesh, Vol. 6. Angiosperms: Dicotyledons (Acanthaceae - Asteraceae). Asiatic Society of Bangladesh, Dhaka, pp. 1408.

Ahmed, Z.U., Hassan, M.A., Begum, Z.N.T., Khondker, M., Kabir, S.M.H., Ahmad, M., Ahmed, A.T.A., Rahman, A.K.A. and Haque, E.U. (Eds) 2009a. Encyclopedia of Flora and Fauna of Bangladesh, Vol. 7. Angiosperms: Dicotyledons (Balsaminaceae - Euphorbiaceae). Asiatic Society of Bangladesh, Dhaka, pp. $1-546$.

Ahmed, Z.U., Hassan, M.A., Begum, Z.N.T., Khondker, M., Kabir, S.M.H., Ahmad, M., Ahmed, A.T.A., Rahman, A.K.A. and Haque, E.U. (Eds) 2009b. Encyclopedia of Flora and Fauna of Bangladesh, Vol. 8. Angiosperms: Dicotyledons (Fabaceae - Lythraceae). Asiatic Society of Bangladesh, Dhaka, pp. 1-478.

Ahmed, Z.U., Hassan, M.A., Begum, Z.N.T., Khondker, M., Kabir, S.M.H., Ahmad, M. and Ahmed, A.T.A. (Eds) 2009c. Encyclopedia of Flora and Fauna of Bangladesh, Vol. 9. Angiosperms: Dicotyledons (Magnoliaceae - Punicaceae). Asiatic Society of Bangladesh, Dhaka, pp. 1-488.

Ahmed, Z.U., Hassan, M.A., Begum, Z.N.T., Khondker, M., Kabir, S.M.H., Ahmad, M., and Ahmed, A.T.A. (Eds) 2009d. Encyclopedia of Flora and Fauna of Bangladesh, Vol. 10. Angiosperms: Dicotyledons (Ranunculaceae - Zygophyllaceae). Asiatic Society of Bangladesh, Dhaka, pp. 1-580.

APG IV. 2016. An update of the Angiosperm Phylogeny Group classification for the orders and families of flowering plants: APG IV. Bot. J. Linn. Soc. 181: 1-20.

Das, D.K. and Alam, M.K. 2001. Trees of Bangladesh. Bangladesh Forest Research Institute, Chittagong, pp. $1-342$.

Dey, C.K., Rahman, M.A. and Wilcock, C.C. 1999. An enumeration of Tree species of Chittagong. Biodiver. Bull. Bangladesh 1: 1-81.

Heinig, R.L. 1925. List of Plants of Chittagong Collectorate and Hill Tracts. Darjeeling, India, pp. 1-84.

Hossain, M.K. 2015. Protected Area Management Plan for Hazarikhil Wildlife Sanctuary (HWS) and Baraiyadhala National Park (BNP) for 2015-2025. Bangladesh Forest Department, Ministry of Environment and Forests, Government of the People's Republic of Bangladesh, pp. 1-109.

Huq, A.M. 1986. Plant Names of Bangladesh. Bangladesh National Herbarium, Dhaka, pp. 1-289.

Nguyen, H.T. 2010. Cycas pectinata. The IUCN Red List of Threatened Species 2010: e.T42062A10617695. http://dx.doi.org/10.2305/IUCN.UK.20103.RLTS.T42062A10617695.en. Accessed on 20 August 2018.

Prain, D. 1903 (reprint 1963). Bengal Plants. Vols. 1 \& 2. Botanical Survey of India, Bishen Singh Mahendra Pal Singh, Dehra Dun, India, pp. 1-1013.

Rahman, M.A. (Ed.). 2013. IUCN Red List Categories of Plants: Red Data Book of Flowering Plants of Bangladesh. Vol. 1. Published by Editor, Chittagong, pp. 1-256.

Rahman, M.A. 2017. Plant diversity in Hazarikhil Wildlife Sanctuary of Chittagong and its Conservation Management. J. Biodivers. Conserv. Bioresour. Manage. 3(2): 43-56.

Rashid, M.E. and Rahman, M.A. 2011. Updated nomenclature and taxonomic status of the plants of Bangladesh included in Hook. f., The Flora of British India: Volume I. Bangladesh J. Plant Taxon. 18(2): 177-197.

Rashid, M.E. and Rahman, M.A. 2012. Updated nomenclature and taxonomic status of the plants of Bangladesh included in Hook. f., The Flora of British India: Volume II. Bangladesh J. Plant Taxon. 19(2): 173-190.

The Plant List 2013. Version 1.1. Published on the Internet; http://www.theplantlist.org/ (Accessed on August 2018). 